Алгебра и анализ

Том. 18 (2006), № 1
St. Petersburg Math. J. Vol. 18 (2007), No. 1, Pages 105-118 S 1061-0022(06)00945-9

Article electronically published on November 27, 2006

\title{
PRODUCTS OF TOEPLITZ OPERATORS ON THE BERGMAN SPACES $A_{\alpha}^{2}$
}

\author{
S. POTT AND E. STROUSE
}

\begin{abstract}
We give a sufficient and a necessary condition for the product of Toeplitz operators $T_{f}^{\alpha} T_{\bar{g}}^{\alpha}$, with $f, g$ analytic, to be bounded on the weighted Bergman space $L_{a}^{2}\left(\mathbb{D},\left(1-|z|^{2}\right)^{\alpha} d A\right)$. We also show that the only compact product of weighted Toeplitz operators is the trivial one.
\end{abstract}

\section{§1. INTRODUCTION}

Let $d A$ denote Lebesgue area measure on the unit disk $\mathbb{D}$, normalized so that the measure of $\mathbb{D}$ equals 1 . For $\alpha>-1$, we denote by $d A_{\alpha}$ the measure $d A_{\alpha}(z)=$ $(\alpha+1)\left(1-|z|^{2}\right)^{\alpha} d A(z)$. For $1 \leq p<+\infty$ the space $L^{p}\left(\mathbb{D}, d A_{\alpha}\right)$ is a Banach space. The (weighted) Bergman space $A_{\alpha}^{2}$ is the closed subspace of analytic functions in the Hilbert space $L^{2}\left(\mathbb{D}, d A_{\alpha}\right)$. We write $\langle\cdot, \cdot\rangle_{\alpha}$ for the inner product on $L^{2}\left(\mathbb{D}, d A_{\alpha}\right)$.

Let $P^{\alpha}$ denote the orthogonal projection from $L^{2}\left(\mathbb{D}, d A_{\alpha}\right)$ onto $A_{\alpha}^{2}$. For each $f$ in $L^{2}\left(\mathbb{D}, d A_{\alpha}\right)$, we have a densely defined Toeplitz operator with symbol $f$, given by

$$
T_{f}^{\alpha}(u)=P^{\alpha}(f u) .
$$

If the function $f$ is analytic (or even harmonic), then it is easy to see that the Toeplitz operator $T_{f}^{\alpha}$ is bounded if and only if $f$ is an essentially bounded function on $\mathbb{D}$. In general, the boundedness of $f$ is easily seen to be sufficient to guarantee the boundedness of the operator $T_{f}^{\alpha}$, but it is not, in fact, always necessary (see $₫ 2$ ).

We shall consider here the question of when, for analytic functions $f$ and $g$, the product $T_{f}^{\alpha} T_{\bar{g}}^{\alpha}$ extends to a bounded linear operator on $A_{\alpha}^{2}$.

This question is motivated by two conjectures of Sarason [S] (see \$2) and by results of Stroethoff and Zheng [StZh1] (see Theorem 2.1 in this paper) and of Nazarov [N1. We shall see that the results of Stroethoff and Zheng can be extended to (and perhaps be more naturally understood in the context of) the weighted Bergman spaces $A_{\alpha}^{2}, \alpha>-1$. The weighted Bergman spaces $A_{\alpha}^{2}$ provide a natural setting for a simultaneous generalization of Sarason's two conjectures.

The second motivation for this paper is, more generally, the idea of the "reproducing kernel thesis" HaNik, namely, the question whether certain classes of operators on reproducing kernel Hilbert spaces can be understood by studying their behavior on the reproducing kernel. Our Berezin transform condition can be understood as such a reproducing kernel condition.

2000 Mathematics Subject Classification. Primary 47B35, 32A36.

Key words and phrases. Weighted Bergman spaces, Toeplitz operators, reproducing kernel thesis.

This work was supported by the European Network on Analysis and Operators (HPRN CT 2000 00116), by a grant by the Nuffield Foundation, and by EPSRC. The first author would like to thank the Departement de Mathématiques Pures, Université Bordeaux I, for their hospitality. 


\section{§2. Principal RESUlts AND Their motivation}

It is well known that the space $A_{\alpha}^{2}$ is a reproducing kernel Hilbert space on $\mathbb{D}$, with reproducing kernels

$$
k_{z}^{\alpha}(w)=\frac{1}{(1-w \bar{z})^{2+\alpha}}
$$

that is,

$$
f(z)=\left\langle f, k_{z}^{\alpha}\right\rangle_{\alpha} \quad\left(z \in \mathbb{D}, f \in A_{\alpha}^{2}\right) .
$$

Let $P^{\alpha}$ denote the orthogonal projection from $L^{2}\left(\mathbb{D}, d A_{\alpha}\right)$ onto $A_{\alpha}^{2}$. Then

$$
\left(P^{\alpha} f\right)(z)=\left\langle f, k_{z}^{\alpha}\right\rangle_{\alpha}=\int_{\mathbb{D}} f \bar{k}_{z}^{\alpha} d A_{\alpha} \quad\left(z \in \mathbb{D}, f \in A_{\alpha}^{2}\right) .
$$

Thus, if $g \in L^{2}\left(\mathbb{D}, d A_{\alpha}\right)$ is such that the Toeplitz operator $T_{g}^{\alpha}$ defined in $\oiint_{1}$ is bounded, then

$$
T_{g}^{\alpha}(u)(w)=\int_{\mathbb{D}} \frac{g(z) u(z)}{(1-w \bar{z})^{2+\alpha}} d A_{\alpha}(z) .
$$

In fact, for any $g \in L^{2}\left(\mathbb{D}, d A_{\alpha}\right)$ and $u \in A_{\alpha}^{2}$, the function $T_{g}^{\alpha}(u)$ thus defined is a well-defined analytic function on the unit disk.

But so far, there seems to exist no satisfactory characterization of those $g$ in $L^{2}\left(\mathbb{D}, d A_{\alpha}\right)$ for which the operator defined in (11) is bounded.

However, there are several partial results, for which we need to introduce some notation. For $\alpha>-1$ and $f \in L^{2}\left(\mathbb{D}, d A_{\alpha}\right)$, let $B_{\alpha}$ denote the (weighted) Berezin transform of $f$, which is defined by

$$
B_{\alpha} f(w)=\left\langle f \tilde{k_{w}^{\alpha}}, \tilde{k_{w}^{\alpha}}\right\rangle_{\alpha}=\int_{\mathbb{D}} \frac{\left(1-|w|^{2}\right)^{\alpha+2}}{|1-w \bar{z}|^{4+2 \alpha}} f(z) d A_{\alpha}(z) \quad(w \in \mathbb{D}) .
$$

If $f$ is a positive function, then the operator $T_{f}^{\alpha}$ defined in (1) is bounded if and only if the function $B_{\alpha}(f)$ is. This result is not true for general $f \in L^{2}\left(\mathbb{D}, d A_{\alpha}\right)$. Indeed, F. Nazarov has shown that there exist functions in $A_{0}^{2}$ such that

$$
\sup _{z \in \mathbb{D}}\left\|T_{f}^{0} \tilde{k_{z}^{0}}\right\|<\infty
$$

but $T_{f}^{0}$ is not a bounded operator $[\mathrm{N} 2$.

Our work here follows the attempt to understand the boundedness of Toeplitz operators via the boundedness of their Berezin transforms. We find a relationship between the boundedness of the product of two Toeplitz operators $T_{f}^{\alpha}$ and $T_{\bar{g}}^{\alpha}$ on $A_{\alpha}^{2}$ and the boundedness of the product of the weighted Berezin transforms of $|f|^{2}$ and $|g|^{2}$.

D. Sarason made the following two conjectures concerning products of Toeplitz operators on the Hardy space $H^{2}(\mathbb{T})$ and on the unweighted Bergman space $A^{2}$. For $u, g \in H^{2}(\mathbb{T})$, the Toeplitz operator $T_{\bar{g}}$ acting on $u$ is pointwise defined by

$$
T_{\bar{g}} u(w)=\left\langle\bar{g} u, k_{w}\right\rangle=\int_{\mathbb{T}} \frac{\bar{g}(\xi) u(\xi)}{1-\bar{\xi} w} d \xi \quad(w \in \mathbb{D}),
$$

where $k_{w}(z)=\frac{1}{1-\bar{w} z}$ is the reproducing kernel in $w$ for $H^{2}(\mathbb{T})$. This can in some sense be regarded as the limit case of (1) for $\alpha \rightarrow-1$.

Sarason asked in $[S]$ for which analytic functions $f$ and $g$ on $\mathbb{D}$ does the operator $T_{f} T_{\bar{g}}$ extend to a bounded linear operator on $H^{2}(\mathbb{T})$ and for which analytic $f$ and $g$ does the operator $T_{f}^{0} T_{\bar{g}}^{0}$ extend to a bounded linear operator on $A_{0}^{2}$. 
He conjectured that, in the case of the Hardy space, a necessary condition found by S. Treil, namely

$$
\sup _{w \in \mathbb{D}}\left\langle|f|^{2} \tilde{k}_{w}, \tilde{k}_{w}\right\rangle\left\langle|g|^{2} \tilde{k}_{w}, \tilde{k}_{w}\right\rangle<\infty,
$$

where $\tilde{k}_{w}=\left(1-|w|^{2}\right)^{1 / 2} \frac{1}{1-\bar{w} z}$ denotes the normalized reproducing kernels of $H^{2}(\mathbb{T})$, is also sufficient. This question turned out to have close links with the question of boundedness of the two-weight Hilbert transform on $L^{2}(\mathbb{T})$ TVZh and was recently answered in the negative by F. Nazarov [N1]. D. Zheng gave a slightly improved sufficient condition in $[\mathrm{Zh}]$.

Furthermore, for the case of the unweighted Bergman space, Sarason conjectured that the necessary condition

$$
\sup _{w \in \mathbb{D}}\left\langle|f|^{2} \tilde{k}_{w}^{0}, \tilde{k}_{w}^{0}\right\rangle_{0}\left\langle|g|^{2} \tilde{k}_{w}^{0}, \tilde{k}_{w}^{0}\right\rangle_{0}=\sup _{w \in \mathbb{D}} B_{0}\left(|f|^{2}\right)(w) B_{0}\left(|g|^{2}\right)(w)<\infty
$$

was also sufficient for the boundedness of $T_{f}^{0} T_{\bar{g}}^{0}$ on $A_{0}^{2}$.

This conjecture is still open. However, the following theorem of Stroethoff and Zheng shows that (5) approximates sufficiency for the boundedness of the product of Toeplitz operators in the following sense.

Theorem 2.1 ([StZh1, 5.1 and 5.2]). (1) If $f$ and $g$ are in $A_{0}^{2}$ and $T_{f}^{0} T_{\bar{g}}^{0}$ is bounded, then

$$
\sup _{w \in \mathbb{D}} B_{0}\left(|f|^{2}\right)(w) B_{0}\left(|g|^{2}\right)(w)<\infty .
$$

(2) If $f$ and $g$ are in $A_{0}^{2}$ and there exists $\epsilon>0$ such that

$$
\sup _{w \in \mathbb{D}} B_{0}\left(|f|^{2+\epsilon}\right)(w) B_{0}\left(|g|^{2+\epsilon}\right)(w)<\infty
$$

then $T_{f}^{0} T_{\bar{g}}^{0}$ is bounded.

Here is our generalization of the Stroethoff-Zheng theorem to weighted Bergman spaces. Note that if $\alpha>\gamma$, then $A_{\gamma}^{2} \subset A_{\alpha}^{2}$. Thus, for $f \in A_{\gamma}^{2}, T_{f}^{\alpha}$ is a densely defined operator on $A_{\alpha}^{2}$.

Theorem 2.2. (1) Let $\gamma \in(-1, \infty)$, and let $f, g \in A_{\gamma}^{2}$. If

$$
\sup _{w \in \mathbb{D}} B_{\gamma}\left(|f|^{2}\right)(w) B_{\gamma}\left(|g|^{2}\right)(w)<\infty,
$$

then for each $\alpha>\gamma, T_{f}^{\alpha} T_{\bar{g}}^{\alpha}$ determines a bounded linear operator $A_{\alpha}^{2} \longrightarrow A_{\alpha}^{2}$.

(2) Let $\alpha \in(-1, \infty)$, and let $f, g \in A_{\alpha}^{2}$. If $T_{f}^{\alpha} T_{\bar{g}}^{\alpha}: A_{\alpha}^{2} \longrightarrow A_{\alpha}^{2}$ is bounded, then $\sup _{w \in \mathbb{D}} B_{\alpha}\left(|f|^{2}\right)(w) B_{\alpha}\left(|g|^{2}\right)(w)<\infty$.

As an immediate consequence concerning Sarason's condition (4), we obtain

Corollary 2.3. Let $f, g \in H^{2}(\mathbb{T})$, and suppose that (44) holds. Then for each $\alpha>-1$, $T_{f}^{\alpha} T_{\bar{g}}^{\alpha}$ determines a bounded linear operator on $A_{\alpha}^{2}$.

Here, we have changed the Toeplitz operators together with the weighted Bergman spaces. Another option is to look at the fixed operator $T_{f}^{\gamma} T_{\bar{g}}^{\gamma}$. Then we obtain the following result, which is in some sense opposite to Theorem 2.2 .

Theorem 2.4. Let $\gamma \in(-1, \infty)$, and let $f, g \in A_{\gamma}^{2}$. If

$$
\sup _{w \in \mathbb{D}} B_{\gamma}\left(|f|^{2}\right)(w) B_{\gamma}\left(|g|^{2}\right)(w)<\infty
$$

then

$$
T_{f}^{\gamma} T_{\bar{g}}^{\gamma}: A_{\alpha}^{2} \longrightarrow A_{\alpha}^{2}
$$

is a bounded operator for $\max \{-1, \gamma-1\}<\alpha<\gamma$. 
Remark. For the case where $f=g$, it is easily seen that the condition

$$
\sup _{w \in \mathbb{D}} B_{\alpha}\left(|f|^{2}\right)(w)<\infty
$$

for any $\alpha>-1$ implies the boundedness of $f$, since

$$
\left\|k_{w}\right\|_{\alpha}^{2}|f(w)|=\left|\left\langle k_{w} f, k_{w}\right\rangle_{\alpha}\right| \leq\left\|k_{w}\right\|_{\alpha}\left\|k_{w} f\right\|_{\alpha}=\left\|k_{w}\right\|_{\alpha}^{2}\left(B_{\alpha}\left(|f|^{2}\right)(w)\right)^{1 / 2} .
$$

Remark. It is easy to show that the analog of Zheng and Stroethoff's condition for $\alpha>-1$, namely

$$
\sup _{w \in \mathbb{D}} B_{\alpha}\left(|f|^{2+\varepsilon}\right)(w) B_{\alpha}\left(|g|^{2+\varepsilon}\right)(w)<\infty
$$

for some $\varepsilon>0$, implies that

$$
\sup _{w \in \mathbb{D}} B_{\alpha-\delta}\left(|f|^{2}\right)(w) B_{\alpha-\delta}\left(|g|^{2}\right)(w)<\infty
$$

for some $\delta>0$. Therefore, by Theorem 2.2. Condition (7) implies that $T_{f} T_{\bar{g}}: A_{\alpha}^{2} \rightarrow A_{\alpha}^{2}$ is bounded.

Conversely, (8) implies, e.g., by the Carleson-type inequality (see Theorem A in $\mathrm{Lu}$, p. 86]), that (77) holds for $\varepsilon / 2=\frac{2+\alpha}{2+\alpha-\delta}-1$. In this sense, our Theorem 2.2 is indeed a generalization of Theorem 2.1 to the standard scale of weighted Bergman spaces.

\section{§3. Introductory LEMMAS}

As in StZh1, we begin with a decomposition of the inner product in the space $A_{\alpha}^{2}$.

Lemma 3.1. For $\alpha>-1$ and $F, G \in A_{\alpha}^{2}$ we have

$$
\begin{aligned}
\int_{\mathbb{D}} F(z) \bar{G}(z) d A_{\alpha}(z)= & \frac{\alpha+3}{\alpha+1} \int_{\mathbb{D}}\left(1-|z|^{2}\right)^{2} F(z) \overline{G(z)} d A_{\alpha}(z) \\
& +\frac{1}{(\alpha+2)(\alpha+1)} \int_{\mathbb{D}}\left(1-|z|^{2}\right)^{2} F^{\prime}(z) \overline{G^{\prime}(z)} d A_{\alpha}(z) \\
& +\frac{1}{(\alpha+1)(\alpha+3)} \int_{\mathbb{D}}\left(1-|z|^{2}\right)^{3} F^{\prime}(z) \overline{G^{\prime}(z)} d A_{\alpha}(z) .
\end{aligned}
$$

Proof. Since the weights are radial, the monomials form an orthonormal basis for $A_{\alpha}^{2}$, and it suffices to prove the identity for the monomials. But in this case, the identity follows from elementary properties of the beta function defined by

$$
\beta(m, n)=\frac{\Gamma(m) \Gamma(n)}{\Gamma(m+n)}=\int_{0}^{1} u^{m-1}(1-u)^{n-1} d u
$$

(see, e.g., $[\mathrm{M}]$ ).

Note also that

$$
\left\|z^{n}\right\|_{\alpha}^{2}=(\alpha+1) \beta(n+1, \alpha+1) \quad \text { for } n \in \mathbb{N}_{0}, \alpha>-1 .
$$

Now, let $a$ and $b$ be two real numbers. For $f \in L^{1}(\mathbb{D}), w \in \mathbb{D}$, we define

$$
P^{a, b}(f)(w)=\left(1-|w|^{2}\right)^{a} \int_{\mathbb{D}} \frac{\left(1-|z|^{2}\right)^{b}}{|1-w \bar{z}|^{2+a+b}} f(z) d A(z) .
$$

The theorem below (a special case of, e.g., [HKZ, Theorem 1.9]) will be used frequently in the following calculations.

Theorem 3.2. Suppose $a$ and $b$ are real numbers and $\alpha>-1$. Then, for $1 \leq p<\infty$, $P^{a, b}$ is bounded on $L^{p}\left(\mathbb{D}, d A_{\alpha}\right)$ if and only if $-p a<\alpha+1<p(b+1)$. 


\section{§4. Proof of the first part of Theorem 2.2}

For $\gamma \in(-1, \infty)$ and $\alpha>\gamma$, we establish a relationship between the boundedness of the product $B_{\gamma}\left(|f|^{2}\right)(w) B_{\gamma}\left(|g|^{2}\right)(w)$ and the boundedness of the operator $T_{f}^{\alpha} T_{\bar{g}}^{\alpha}$ acting on the space $A_{\alpha}^{2}$. First, we use the preceding results to get some estimates.

Lemma 4.1. Let $\gamma \in(-1, \infty)$ and $\alpha>\gamma$. Suppose that $f \in L^{2}\left(\mathbb{D}, d A_{\alpha}\right)$. Then for each $h \in A_{\alpha}^{2}$ and $w \in \mathbb{D}$ we have

$$
\left|\left(T \frac{\alpha}{f} h\right)(w)\right| \leq\left(\frac{\alpha+1}{\gamma+1}\right)^{1 / 2} \frac{1}{\left(1-|w|^{2}\right)^{1+\frac{\alpha}{2}}}\left(B_{\gamma}\left(|f|^{2}\right)(w)\right)^{\frac{1}{2}}\|h\|_{\alpha}
$$

Proof. Let $\epsilon=\alpha-\gamma$. First we notice that, for any $u, v \in L^{2}\left(\mathbb{D}, d A_{\gamma}\right)$, we have

$$
\begin{aligned}
\langle u, v\rangle_{\alpha} & =\frac{\alpha+1}{\gamma+1}\left\langle u, v\left(1-|z|^{2}\right)^{\epsilon}\right\rangle_{\gamma}, \\
\|u\|_{\gamma} & =\left(\frac{\gamma+1}{\alpha+1}\right)^{1 / 2}\left\|u\left(1-|z|^{2}\right)^{-\frac{\epsilon}{2}}\right\|_{\alpha}
\end{aligned}
$$

and that

$$
k_{w}^{\alpha}=\frac{1}{(1-\bar{w} z)^{\epsilon}} k_{w}^{\gamma} .
$$

Using (10) and (11), and then dividing by the norm of $k_{w}^{\gamma}$, we see that

$$
\begin{aligned}
\left|\left(T \frac{\alpha}{f} h\right)(w)\right| & =\left\langle h, f k_{w}^{\alpha}\right\rangle_{\alpha} \\
& =\frac{\alpha+1}{\gamma+1}\left\langle h \frac{\left(1-|z|^{2}\right)^{\epsilon}}{(1-w \bar{z})^{\epsilon}}, f k_{w}^{\gamma}\right\rangle_{\gamma} \\
& =\frac{\alpha+1}{\gamma+1} \frac{1}{\left(1-|w|^{2}\right)^{1+\frac{\gamma}{2}}}\left\langle h \frac{\left(1-|z|^{2}\right)^{\epsilon}}{(1-w \bar{z})^{\epsilon}}, f \frac{k_{w}^{\gamma}}{\left\|k_{w}^{\gamma}\right\|}\right\rangle_{\gamma} .
\end{aligned}
$$

Then, using Hölder's inequality, (11), and a little division, we get

$$
\begin{aligned}
\left|\left(T \frac{\alpha}{f} h\right)(w)\right| & \leq \frac{\alpha+1}{\gamma+1} \frac{1}{\left(1-|w|^{2}\right)^{1+\frac{\gamma}{2}}}\left\|h \frac{\left(1-|z|^{2}\right)^{\epsilon}}{(1-w \bar{z})^{\epsilon}}\right\|_{\gamma}\left(B_{\gamma}\left(|f|^{2}\right)(w)\right)^{\frac{1}{2}} \\
& =\frac{\alpha+1}{\gamma+1} \frac{1}{\left(1-|w|^{2}\right)^{1+\frac{\alpha}{2}}}\left\|h \frac{\left(1-|z|^{2}\right)^{\epsilon}\left(1-|w|^{2}\right)^{\frac{\epsilon}{2}}}{(1-w \bar{z})^{\epsilon}}\right\|_{\gamma}\left(B_{\gamma}\left(|f|^{2}\right)(w)\right)^{\frac{1}{2}} \\
& =\left(\frac{\alpha+1}{\gamma+1}\right)^{1 / 2} \frac{1}{\left(1-|w|^{2}\right)^{1+\frac{\alpha}{2}}}\left\|h \frac{\left(1-|z|^{2}\right)^{\frac{\epsilon}{2}}\left(1-|w|^{2}\right)^{\frac{\epsilon}{2}}}{(1-w \bar{z})^{\epsilon}}\right\|_{\alpha}\left(B_{\gamma}\left(|f|^{2}\right)(w)\right)^{\frac{1}{2}} .
\end{aligned}
$$

Now, since

we see that

$$
\frac{\left(1-|z|^{2}\right)^{1 / 2}\left(1-|w|^{2}\right)^{1 / 2}}{|1-\bar{z} w|} \leq 1
$$

$$
\left|\left(T_{\bar{f}}^{\alpha} h\right)(w)\right| \leq\left(\frac{\alpha+1}{\gamma+1}\right)^{1 / 2} \frac{1}{\left(1-|w|^{2}\right)^{1+\frac{\alpha}{2}}}\|h\|_{\alpha}\left(B_{\gamma}\left(|f|^{2}\right)(w)\right)^{\frac{1}{2}} .
$$

Next, we present a bound for the values of the derivative of $T_{\frac{\alpha}{f}}^{\alpha} h$.

Lemma 4.2. Let $\alpha, \gamma \in(-1, \infty)$ with $\alpha>\gamma$ and $\epsilon=\alpha-\gamma$, and let $f \in L^{2}\left(\mathbb{D}, A_{\gamma}\right)$. Then for each $w \in \mathbb{D}$ we have

$$
\left|\left(T \frac{\alpha}{f} h\right)^{\prime}(w)\right| \leq \frac{(\alpha+1)(\alpha+2)}{(\gamma+1)^{1 / 2}} \frac{\left(B_{\gamma}\left(|f|^{2}\right)(w)\right)^{\frac{1}{2}}}{\left(1-|w|^{2}\right)^{1+\frac{\gamma}{2}}}\left(P^{0,2 \epsilon}\left(|h|^{2}\left(1-|z|^{2}\right)^{\gamma}\right)(w)\right)^{\frac{1}{2}} .
$$


Proof. By direct calculation,

$$
\begin{aligned}
\left|\left(T_{\bar{f}}^{\alpha} h\right)^{\prime}(w)\right| & =\left|(2+\alpha) \int_{\mathbb{D}} \frac{\overline{z f}(z) h(z)}{(1-\bar{z} w)^{3+\alpha}} d A_{\alpha}(z)\right| \\
& \leq(\alpha+2) \int_{\mathbb{D}} \frac{|f(z)||h(z)|}{|1-\bar{z} w|^{3+\alpha}} d A_{\alpha}(z) \\
& =\frac{(\alpha+2)(\alpha+1)}{\gamma+1} \int_{\mathbb{D}} \frac{|f(z)||h(z)|\left(1-|z|^{2}\right)^{\epsilon}}{|1-\bar{z} w|^{2+\gamma}|1-\bar{z} w|^{1+\epsilon}} d A_{\gamma}(z) .
\end{aligned}
$$

Now, with an obvious application of Hölder's inequality, we see that

$$
\begin{aligned}
\left|\left(T_{\bar{f}}^{\alpha} h\right)^{\prime}(w)\right| \leq & \frac{(\alpha+1)(\alpha+2)}{\gamma+1}\left(\int_{\mathbb{D}} \frac{|f(z)|^{2}}{|1-\bar{z} w|^{4+2 \gamma}} d A_{\gamma}(z)\right)^{\frac{1}{2}} \\
& \times\left(\int_{\mathbb{D}} \frac{|h(z)|^{2}\left(1-|z|^{2}\right)^{2 \epsilon}}{|1-\bar{z} w|^{2+2 \epsilon}} d A_{\gamma}(z)\right)^{\frac{1}{2}} \\
= & \frac{(\alpha+1)(\alpha+2)}{(\gamma+1)^{1 / 2}} \frac{1}{\left(1-|w|^{2}\right)^{1+\frac{\gamma}{2}}}\left(B_{\gamma}\left(|f|^{2}\right)(w)\right)^{\frac{1}{2}} \\
& \times\left(\int_{\mathbb{D}} \frac{|h(z)|^{2}\left(1-|z|^{2}\right)^{2 \epsilon+\gamma}}{|1-\bar{z} w|^{2+2 \epsilon}} d A(z)\right)^{\frac{1}{2}} \\
= & \frac{(\alpha+1)(\alpha+2)}{(\gamma+1)^{1 / 2}} \frac{1}{\left(1-|w|^{2}\right)^{1+\frac{\gamma}{2}}}\left(B_{\gamma}\left(|f|^{2}\right)(w)\right)^{\frac{1}{2}} \\
& \times\left(P^{0,2 \epsilon}\left(|h|^{2}\left(1-|z|^{2}\right)^{\gamma}\right)(w)\right)^{\frac{1}{2}},
\end{aligned}
$$

which finishes the proof.

Now we are ready to prove the first part of Theorem 2.2 .

Proof of 2.2(1). Let $\gamma>-1$ and $\alpha>\gamma$, and let $u$ and $v$ be in $A_{\alpha}^{2}$. As in [StZh1, we use a decomposition of $\left\langle T_{f}^{\alpha} T_{\bar{g}}^{\alpha} u, v\right\rangle_{\alpha}$ to show that $T_{f}^{\alpha} T_{\bar{g}}^{\alpha}$ is bounded on $A_{\alpha}^{2}$. It follows from Lemma 3.1 that

$$
\left\langle T_{f}^{\alpha} T_{\bar{g}}^{\alpha} u, v\right\rangle_{\alpha}=\left\langle T_{\bar{g}}^{\alpha} u, T \frac{\alpha}{f} v\right\rangle_{\alpha}=\mathrm{I}+\mathrm{II}+\mathrm{III},
$$

where

$$
\begin{aligned}
\mathrm{I} & =\frac{\alpha+3}{\alpha+1} \int_{\mathbb{D}}\left(1-|w|^{2}\right)^{2}\left(T_{\bar{g}}^{\alpha} u\right)(w) \overline{\left(T_{\bar{f}}^{\alpha} v\right)(w)} d A_{\alpha}(w), \\
\mathrm{II} & =\frac{1}{(\alpha+2)(\alpha+1)} \int_{\mathbb{D}}\left(1-|w|^{2}\right)^{2}\left(T_{\bar{g}}^{\alpha} u\right)^{\prime}(w), \overline{\left(T_{\bar{f}}^{\alpha} v\right)^{\prime}(w)} d A_{\alpha}(w), \\
\mathrm{III} & =\frac{1}{(\alpha+1)(\alpha+3)} \int_{\mathbb{D}}\left(1-|w|^{2}\right)^{3}\left(T_{\bar{g}}^{\alpha} u\right)^{\prime}(w) \overline{\left(T_{\bar{f}}^{\alpha}\right)^{\prime}(w)} d A_{\alpha}(w) .
\end{aligned}
$$

Let $M=\sup _{w \in \mathbb{D}} B_{\gamma}\left(|f|^{2}\right)(w) B_{\gamma}\left(|g|^{2}\right)(w)$. Using Lemma 4.1, we obtain

$$
\begin{aligned}
\mathrm{I} & \leq \frac{\alpha+3}{\gamma+1} \int_{\mathbb{D}} \frac{\left(1-|w|^{2}\right)^{2}}{\left(1-|w|^{2}\right)^{2+\alpha}}\left(( B _ { \gamma } ( | f | ^ { 2 } ) ( w ) ) ^ { \frac { 1 } { 2 } } \left(\left(B_{\gamma}\left(|g|^{2}\right)(w)\right)^{\frac{1}{2}}\|u\|_{\alpha}\|v\|_{\alpha} d A_{\alpha}(w)\right.\right. \\
& \leq \frac{(\alpha+1)(\alpha+3)}{(\gamma+1)} \int_{\mathbb{D}}\left(( B _ { \gamma } ( | f | ^ { 2 } ) ( w ) ) ^ { \frac { 1 } { 2 } } \left(\left(B_{\gamma}\left(|g|^{2}\right)(w)\right)^{\frac{1}{2}}\|u\|_{\alpha}\|v\|_{\alpha} d A(w)\right.\right. \\
& \leq \frac{(\alpha+1)(\alpha+3)}{(\gamma+1)} M^{\frac{1}{2}}\|u\|_{\alpha}\|v\|_{\alpha},
\end{aligned}
$$

which takes care of the first term. 
As for the second term, by Lemma 4.2, once again setting $\epsilon=\alpha-\gamma$, we have

$$
\begin{aligned}
\mathrm{II} \leq & \frac{(\alpha+1)(\alpha+2)}{\gamma+1} \\
& \times \int_{\mathbb{D}} \frac{1}{\left(1-|w|^{2}\right)^{\gamma}}\left(B_{\gamma}\left(|f|^{2}\right)(w)\right)^{\frac{1}{2}}\left(B_{\gamma}\left(|g|^{2}\right)(w)\right)^{\frac{1}{2}} \\
& \quad \times\left(P^{0,2 \epsilon}\left(|u|^{2}\left(1-|z|^{2}\right)^{\gamma}\right)(w)\right)^{\frac{1}{2}}\left(P^{0,2 \epsilon}\left(|v|^{2}\left(1-|z|^{2}\right)^{\gamma}\right)(w)\right)^{\frac{1}{2}} d A_{\alpha}(w) \\
\leq & M^{1 / 2} \frac{(\alpha+1)(\alpha+2)}{\gamma+1} \\
& \times \int_{\mathbb{D}} \frac{1}{\left(1-|w|^{2}\right)^{\gamma}}\left(P^{0,2 \epsilon}\left(|u|^{2}\left(1-|z|^{2}\right)^{\gamma}\right)(w)\right)^{\frac{1}{2}}\left(P^{0,2 \epsilon}\left(|v|^{2}\left(1-|z|^{2}\right)^{\gamma}\right)(w)\right)^{\frac{1}{2}} d A_{\alpha}(w) \\
= & M^{1 / 2} \frac{(\alpha+1)^{2}(\alpha+2)}{\gamma+1} \\
& \times \int_{\mathbb{D}}\left(1-|w|^{2}\right)^{\epsilon}\left(P^{0,2 \epsilon}\left(|u|^{2}\left(1-|z|^{2}\right)^{\gamma}\right)(w)\right)^{\frac{1}{2}}\left(P^{0,2 \epsilon}\left(|v|^{2}\left(1-|z|^{2}\right)^{\gamma}\right)(w)\right)^{\frac{1}{2}} d A(w) .
\end{aligned}
$$

So, applying Hölder's inequality, we obtain

$$
\begin{aligned}
\mathrm{II} \leq & M^{\frac{1}{2}} \frac{(\alpha+1)^{2}(\alpha+2)}{(\gamma+1)(\alpha-\gamma+1)}\left(\int_{\mathbb{D}} P^{0,2 \epsilon}\left(|u|^{2}\left(1-|z|^{2}\right)^{\gamma}(w) d A_{\epsilon}(w)\right)^{\frac{1}{2}}\right. \\
& \times\left(\int_{\mathbb{D}} P^{0,2 \epsilon}\left(|v|^{2}\left(1-|z|^{2}\right)^{\gamma}(w) d A_{\epsilon}(w)\right)^{\frac{1}{2}} .\right.
\end{aligned}
$$

But, by Theorem 3.2, the operator $P^{0,2 \epsilon}$ is bounded on $L^{1}\left(\mathbb{D}, d A_{\epsilon}\right)$, and so

$$
\begin{aligned}
\int_{\mathbb{D}} P^{0,2 \epsilon}\left(|u|^{2}\left(1-|z|^{2}\right)^{\gamma}\right)(w) d A_{\epsilon}(w) \\
\quad \leq\left\|P^{0,2 \epsilon}\right\|_{L^{1}\left(\mathbb{D}, d A_{\epsilon}\right)} \int_{\mathbb{D}}\left(|u|^{2}\left(1-|z|^{2}\right)^{\gamma}\right)(w) d A_{\epsilon}(w) \\
\quad=\frac{\alpha-\gamma+1}{(\alpha+1)^{1 / 2}}\left\|P^{0,2 \epsilon}\right\|_{L^{1}\left(\mathbb{D}, d A_{\epsilon}\right)}\|u\|_{\alpha}
\end{aligned}
$$

and

Thus,

$$
\int_{\mathbb{D}} P^{0,2 \epsilon}\left(|v|^{2}\left(1-|z|^{2}\right)^{\gamma}\right)(w) d A_{\epsilon}(w) \leq \frac{\alpha-\gamma+1}{(\alpha+1)^{1 / 2}}\left\|P^{0,2 \epsilon}\right\|_{L^{1}\left(\mathbb{D}, d A_{\epsilon}\right)}\|v\|_{\alpha} .
$$

$$
\mathrm{II} \leq M^{\frac{1}{2}} \frac{(\alpha+1)(\alpha+2)}{\gamma+1}\left\|P^{0,2 \epsilon}\right\|_{L^{1}\left(\mathbb{D}, d A_{\epsilon}\right)}\|v\|_{\alpha}\|u\|_{\alpha} .
$$

An analogous estimate for III follows easily. This makes it clear that there exists a constant $K$ such that

and the proof is finished.

$$
\left|\left\langle T_{f}^{\alpha} T_{\bar{g}}^{\alpha} u, v\right\rangle_{\alpha}\right| \leq K\|v\|_{\alpha}\|u\|_{\alpha}
$$

\section{§5. The SECOND PART of TheOrem 2.2}

To prove the second part of Theorem 2.2, we first introduce some notation. Let $\mathcal{H}$ be a Hilbert space, and let $T \in \mathcal{L}(\mathcal{H})$. Then we can define the bounded linear operator

$$
M_{T}: \mathcal{L}(\mathcal{H}) \rightarrow \mathcal{L}(\mathcal{H}), \quad A \mapsto T^{*} A T
$$

(see, e.g., VMü ). If $T$ is a contraction, then so is $M_{T}$. In this case, we can observe that the Taylor expansion of the function $x \mapsto(1-x)^{\alpha+2}$ around $0,(1-x)^{\alpha+2}=\sum_{k=0}^{\infty} \gamma_{\alpha, k} x^{k}$, 
is absolutely convergent on the closed unit disk in $\mathbb{C}$ for $\alpha \geq-1$, and we can use the obvious functional calculus to define the operator $\left(1-M_{T}\right)^{\alpha+2}$ by the equation

$$
\left(1-M_{T}\right)^{\alpha+2}(A)=\sum_{k=0}^{\infty} \gamma_{\alpha, k}\left(T^{*}\right)^{k} A(T)^{k} .
$$

Now, let $\alpha>-1$, and let $\left(e_{n}\right)_{n \in \mathbb{N}_{0}}$ denote the standard orthonormal basis of $A_{\alpha}^{2}$, which consists of normalized monomials.

Lemma 5.1. We have

$$
e_{n}=\beta_{\alpha, n}^{1 / 2} z^{n}
$$

where the $\beta_{\alpha, n}$ are the Taylor coefficients of the function $x \mapsto(1-x)^{-2-\alpha}$ around 0 .

Proof. Integration by parts shows that $\left\|z^{n}\right\|_{A_{\alpha}}^{2}=\frac{n}{\alpha+2}\left\|z^{n-1}\right\|_{A_{\alpha+1}}^{2}$, and differentiation shows that

$$
\begin{aligned}
\beta_{\alpha, n}^{2} & =\left.\frac{1}{n !}\left(\frac{d}{d x}\right)^{n}(1-x)^{-\alpha-2}\right|_{x=0} \\
& =\left.\frac{1}{n !}(\alpha+2)\left(\frac{d}{d x}\right)^{n-1}(1-x)^{-(\alpha+1)-2}\right|_{x=0}=\frac{\alpha+2}{n} \beta_{\alpha+1, n-1} .
\end{aligned}
$$

The remainder follows by induction.

For $e, f \in A_{\alpha}^{2}$, let $e \otimes f$ denote the rank one operator given by $h \mapsto\langle h, f\rangle e$. Let 1 denote the identity operator on $A_{\alpha}^{2}$.

Theorem 5.2. Let $\alpha>-1$. Then

$$
\left(1-M_{T_{\bar{z}}^{\alpha}}\right)^{\alpha+2}(\mathbf{1})=e_{0} \otimes e_{0} .
$$

Proof. We can write $\mathbf{1}=\sum_{n=0}^{\infty} \beta_{\alpha, n} z^{n} \otimes z^{n}$ in the sense of a series convergent in the strong operator topology. Thus for $h \in A_{\alpha}^{2}$,

$$
\begin{gathered}
\left\langle\left(1-M_{T_{\bar{z}}^{\alpha}}\right)^{\alpha+2}(\mathbf{1}) h, h\right\rangle_{\alpha}=\sum_{k=0}^{\infty} \gamma_{\alpha, k}\left\langle\left(T_{z}^{\alpha}\right)^{k}\left(\sum_{n=0}^{\infty} \beta_{\alpha, n} z^{n} \otimes z^{n}\right)\left(T_{\bar{z}}^{\alpha}\right)^{k} h, h\right\rangle_{\alpha} \\
=\sum_{k=0}^{\infty} \sum_{n=0}^{\infty} \gamma_{\alpha, k} \beta_{\alpha, n}\left\langle z^{n+k} \otimes z^{n+k} h, h\right\rangle_{\alpha}=\left\langle e_{0} \otimes e_{0} h, h\right\rangle_{\alpha} .
\end{gathered}
$$

As usual, we let $\phi_{w}$ denote the Möbius transformation $\mathbb{D} \rightarrow \mathbb{D}, z \mapsto \frac{w-z}{1-\bar{w} z}$. We define the operator $U_{w}^{\alpha}$ by

$$
U_{w}^{\alpha}: A_{\alpha}^{2} \rightarrow A_{\alpha}^{2}, \quad h \mapsto\left(h \circ \phi_{w}\right) \tilde{k}_{w}^{\alpha}
$$

Using the facts that

$$
\tilde{k_{w}^{\alpha}}\left(\phi_{w}(z)\right)=\frac{1}{\tilde{k_{w}^{\alpha}}(z)}
$$

and that

$$
\int_{\mathbb{D}} f\left(\phi_{w}(z)\right) d A_{\alpha}(z)=\int_{\mathbb{D}} f(z)\left|\tilde{k_{w}^{\alpha}}(z)\right|^{2} d A_{\alpha}(z),
$$

it is easy to see that $U_{w}^{\alpha}$ is unitary and that we have the intertwining relation

$$
U_{w}^{\alpha} T_{f}^{\alpha}=T_{f \circ \phi_{w}}^{\alpha} U_{w}^{\alpha}
$$

for a Toeplitz operator with analytic symbol $f$. This also means that

$$
T_{\bar{g}}^{\alpha}\left(U_{w}^{\alpha}\right)^{*}=\left(U_{w}^{\alpha}\right)^{*} T \frac{\alpha}{g \circ \phi_{w}}
$$

for a Toeplitz operator with antianalytic symbol $\bar{g}$. 
Lemma 5.3. We have

$$
\tilde{k}_{w}^{\alpha} \otimes \tilde{k}_{w}^{\alpha}=\left(1-M_{T_{\phi_{w}}}\right)^{\alpha+2}(\mathbf{1})
$$

Proof.

$$
\begin{aligned}
\tilde{k}_{w}^{\alpha} \otimes \tilde{k}_{w}^{\alpha} & =\left(U_{w}^{\alpha} e_{0}\right) \otimes\left(U_{w}^{\alpha} e_{0}\right)=U_{w}^{\alpha}\left(e_{0} \otimes e_{0}\right) U_{w}^{\alpha *} \\
& =U_{w}^{\alpha}\left(\sum_{k=0}^{\infty} \gamma_{\alpha, k} T_{z}^{\alpha k} T_{\bar{z}}^{\alpha k}\right) U_{w}^{\alpha *}=\sum_{k=0}^{\infty} \gamma_{\alpha, k} T_{\phi_{w}}^{\alpha{ }^{k}} T_{\bar{\phi}_{w}}^{\alpha{ }^{k}}=\left(1-M_{T_{\phi_{w}}}\right)^{\alpha+2}(\mathbf{1}) .
\end{aligned}
$$

Lemma 5.4. If $T_{f}^{\alpha} T_{\bar{g}}^{\alpha}$ determines a bounded linear operator on $A_{\alpha}^{2}$, then $\left(T_{f} \tilde{k}_{w}^{\alpha}\right) \otimes\left(T_{g} \tilde{k}_{w}^{\alpha}\right)$ is also bounded, and there exists an absolute constant $K>0$ such that

$$
\left\|\left(T_{f}^{\alpha} \tilde{k}_{w}^{\alpha}\right) \otimes\left(T_{g}^{\alpha} \tilde{k}_{w}^{\alpha}\right)\right\| \leq K\left\|T_{f}^{\alpha} T_{\bar{g}}^{\alpha}\right\| .
$$

Proof. Using commutativity of analytic Toeplitz operators, we find

$$
\begin{aligned}
\left(T_{f}^{\alpha} \tilde{k}_{w}^{\alpha}\right) \otimes\left(T_{g}^{\alpha} \tilde{k}_{w}^{\alpha}\right) & =\sum_{k=0}^{\infty} \gamma_{\alpha, k} T_{f}^{\alpha}\left(T_{\phi_{w}}^{\alpha}\right)^{k}\left(T_{\bar{\phi}_{w}}^{\alpha}\right)^{k} T_{\bar{g}}^{\alpha} \\
& =\sum_{k=0}^{\infty} \gamma_{\alpha, k}\left(T_{\phi_{w}}^{\alpha}\right)^{k} T_{f}^{\alpha} T_{\bar{g}}^{\alpha}\left(T_{\bar{\phi}_{w}}^{\alpha}\right)^{k} \\
& =\left(1-M_{T_{\phi_{w}}^{\alpha}}\right)^{\alpha+2}\left(T_{f}^{\alpha} T_{\bar{g}}^{\alpha}\right) .
\end{aligned}
$$

Since $M_{T_{\phi w}^{\alpha}}$ is a contraction on $\mathcal{L}\left(A_{\alpha}^{2}\right)$ and $(1-x)^{\alpha+2}$ has an absolutely convergent power series expansion on the closed unit disk, it follows that $\left(1-M_{T_{\phi_{w}}^{\alpha}}\right)^{\alpha+2}$ is a bounded operator on $\mathcal{L}\left(A_{\alpha}^{2}\right)$, and therefore, letting $K=\sum_{k=0}^{\infty}\left|\gamma_{\alpha, k}\right|$, we have

$$
\left\|\left(1-M_{T_{\phi_{w}}^{\alpha}}\right)^{\alpha+2}\left(T_{f}^{\alpha} T_{\bar{g}}^{\alpha}\right)\right\| \leq K\left\|T_{f}^{\alpha} T_{\bar{g}}^{\alpha}\right\| .
$$

Proof of Theorem $2.2(2)$. The reverse direction of Theorem 2.2 is an easy consequence of Lemma 5.4 because if $f, g \in A_{\alpha}^{2}$ and $w \in \mathbb{D}$, then

$$
\begin{aligned}
& \left(B_{\alpha}\left(|f|^{2}\right)(w)\right)^{1 / 2}\left(B_{\alpha}\left(|g|^{2}\right)(w)\right)^{1 / 2}=\left\|f \tilde{k}_{w}^{\alpha}\right\|_{\alpha}\left\|g \tilde{k}_{w}^{\alpha}\right\|_{\alpha} \\
& \quad=\left\|T_{f}^{\alpha} \tilde{k}_{w}^{\alpha}\right\|_{\alpha}\left\|T_{g}^{\alpha} \tilde{k}_{w}^{\alpha}\right\|_{\alpha}=\left\|\left(T_{f}^{\alpha} \tilde{k}_{w}^{\alpha}\right) \otimes\left(T_{g}^{\alpha} \tilde{k}_{w}^{\alpha}\right)\right\| \leq K\left\|T_{f}^{\alpha} T_{\bar{g}}^{\alpha}\right\| .
\end{aligned}
$$

Formally inverting identity (17), we obtain the following description of $T_{f}^{\alpha} T_{\bar{g}}^{\alpha}$ on $A_{\alpha}^{2}$. As before, let $\beta_{\alpha, k}$ denote the $k$ th Taylor coefficient of $x \mapsto(1-x)^{-\alpha-2}$ at $x=0$.

Proposition 5.5. Let $f, g \in A_{\alpha}^{2}$. $T_{f}^{\alpha} T_{\bar{g}}^{\alpha}$ determines a bounded linear operator on $A_{\alpha}^{2}$ if and only if the series

$$
\sum_{k=0}^{\infty} \beta_{\alpha, k} f \phi_{w}^{k} \tilde{k}_{w}^{\alpha} \otimes g \phi_{w}^{k} \tilde{k}_{w}^{\alpha}
$$

converges in the strong operator topology of $\mathcal{L}\left(A_{\alpha}^{2}\right)$ for some (and equivalently, for all) $w \in \mathbb{D}$. In this case,

$$
T_{f}^{\alpha} T_{\bar{g}}^{\alpha}=\sum_{k=0}^{\infty} \beta_{\alpha, k} f \phi_{w}^{k} \tilde{k}_{w}^{\alpha} \otimes g \phi_{w}^{k} \tilde{k}_{w}^{\alpha}=\sum_{k=0}^{\infty} \beta_{\alpha, k} f z^{k} \otimes g z^{k} \quad \text { for all } w \in \mathbb{D} .
$$

Proof. Suppose that $T_{f}^{\alpha} T_{\bar{g}}^{\alpha}$ determines a bounded linear operator on $A_{\alpha}^{2}$. Since, by Lemma 5.1. $\left(\beta_{\alpha, k}^{\frac{1}{2}} \phi_{w}^{k} \tilde{k}_{w}^{\alpha}\right)_{k \in \mathbb{N}_{0}}=\left(U_{w}^{\alpha} \beta_{\alpha, k}^{\frac{1}{2}} z^{k}\right)_{k \in \mathbb{N}_{0}}$ is an orthonormal basis of $A_{\alpha}^{2}$ for each 
$w \in \mathbb{D}$, we see that

$$
\begin{aligned}
T_{f} T_{\bar{g}} h & =T_{f} \sum_{k=0}^{\infty} \beta_{\alpha, k}\left(z^{k} \otimes z^{k}\right) T_{\bar{g}} h \\
& =\sum_{k=0}^{\infty} \beta_{\alpha, k}\left(f z^{k} \otimes g z^{k}\right) h=\sum_{k=0}^{\infty} \beta_{\alpha, k}\left(f \phi_{w}^{k} \tilde{k}_{w}^{\alpha} \otimes g \phi_{w}^{k} \tilde{k}_{w}^{\alpha}\right) h
\end{aligned}
$$

for each $h$ in a dense subset of $A_{\alpha}^{2}$ and for each $w \in \mathbb{D}$. Conversely, the convergence of the last sum in (18) for each $h \in A_{\alpha}^{2}$ and some fixed $w \in \mathbb{D}$ implies the boundedness of $T_{f} T_{\bar{g}}$ by the Banach-Steinhaus theorem.

We proceed to the proof of Corollary 2.3. Note that, as in (13),

$$
U_{w}: H^{2}(\mathbb{T}) \rightarrow H^{2}(\mathbb{T}), \quad h \mapsto\left(h \circ \phi_{w}\right) \tilde{k}_{w}
$$

determines a unitary operator on $H^{2}(\mathbb{T})$ for each $w \in \mathbb{D}$, which satisfies the intertwining relation

$$
U_{w} T_{f}=T_{f \circ \phi_{w}} U_{w}
$$

for each analytic $f$.

Proof of Corollary 2.3. It suffices to show that

$$
B_{\alpha}\left(|f|^{2}\right)(w) \leq\left\langle|f|^{2} \tilde{k}_{w}, \tilde{k}_{w}\right\rangle=\left\|T_{f} \tilde{k}_{w}\right\|^{2}
$$

for all $\alpha>-1, f \in H^{2}(\mathbb{T}), w \in \mathbb{D}$, since in this case condition (4) implies

$$
\sup _{w \in \mathbb{D}} B_{\alpha}\left(|f|^{2}\right)(w) B_{\alpha}\left(|g|^{2}\right)(w)<\infty
$$

for all $\alpha>-1$. Since

$$
B_{\alpha}\left(|f|^{2}\right)(w)=\left\|T_{f}^{\alpha} \tilde{k}_{w}^{\alpha}\right\|_{\alpha}^{2}=\left\|T_{f \circ \phi_{w}}^{\alpha} k_{0}^{\alpha}\right\|_{\alpha}^{2}=B_{\alpha}\left(\left|f \circ \phi_{w}\right|^{2}\right)(0)
$$

and

$$
\left\langle|f|^{2} \tilde{k}_{w}, \tilde{k}_{w}\right\rangle=\left\|T_{f} \tilde{k}_{w}\right\|^{2}=\left\|T_{f \circ \phi_{w}} k_{0}\right\|^{2}
$$

for all $w \in \mathbb{D}, f \in H^{2}$, by the intertwining relations (16) and (19), it suffices to check (20) for $w=0$, in which case $\tilde{k}_{w}^{\alpha}$ and $\tilde{k}_{w}$ are constantly 1 . Relation (20) now follows simply from the contractive embedding $H^{2}(\mathbb{T}) \subset A_{\alpha}^{2}$.

Finally, as in StZh1, we can use our results to show that the only compact product of Toeplitz operators on a weighted Bergman space is the trivial one. First, we need the following lemma.

Lemma 5.6. Let $A$ be a compact operator on $A_{\alpha}^{2}$, and suppose that $w_{n} \rightarrow \eta$, where $\eta$ is a point on the unit circle $\mathbb{T}$. Then

$$
\lim _{n \rightarrow \infty}\left\|\left(1-M_{T_{\phi_{w_{n}}}^{\alpha}}\right)^{\alpha+2}(A)\right\|_{\alpha}=0 .
$$

Proof. Using exactly the same argument as in the proof of [StZh1, Lemma 6.1], we show that if $A$ is any compact operator on $A_{\alpha}^{2}$, then

$$
\left\|M_{T_{\phi_{w_{n}}}^{\alpha}}(A)-A\right\|_{\alpha} \rightarrow 0
$$

as $w_{n} \rightarrow \eta \in \mathbb{T}$. Now, using (22), we easily see that

$$
\left\|M_{T_{\phi_{w_{n}}}^{\alpha}}^{k}(A)-A\right\|_{\alpha} \rightarrow 0
$$


for each compact $A$ and each $k \in \mathbb{N}$. So, writing

$$
\begin{aligned}
\left\|\left(1-M_{T_{\phi_{w_{n}}}^{\alpha}}\right)^{\alpha+2}(A)\right\|_{\alpha} & =\left\|\left(\mathbf{1}+\sum_{k=1}^{\infty}(-1)^{k} \frac{(\alpha+2) \cdots(\alpha-k+3)}{k !} M_{T_{\phi_{w_{n}}}^{\alpha}}^{k}\right)(A)\right\|_{\alpha} \\
& \left.=\| \sum_{k=1}^{\infty}(-1)^{k} \frac{(\alpha+2) \cdots(\alpha-k+3)}{k !}\left(M_{T_{\phi_{w_{n}}}^{\alpha}}^{k}\right)(A)-A\right) \|_{\alpha} \\
& \leq \sum_{k=1}^{\infty}(-1)^{k} \frac{(\alpha+2) \cdots(\alpha-k+3)}{k !}\left\|\left(M_{T_{\phi_{w}}^{\alpha}}^{k}\right)(A)-A\right\|_{\alpha},
\end{aligned}
$$

we see that the lemma follows from $(23)$, the summability of the series $\sum_{k=1}^{\infty} \frac{(\alpha+2) \cdots(\alpha-k+3)}{k !}$,
and the dominated convergence theorem.

Proposition 5.7. Let $f, g \in A_{\alpha}^{2}$. Then $T_{f}^{\alpha} T_{\bar{g}}^{\alpha}$ extends to a compact operator on $A_{\alpha}^{2}$ if and only if $f=0$ or $g=0$.

Proof. Suppose that $T_{f}^{\alpha} T_{\bar{g}}^{\alpha}$ extends to a compact operator on $A_{\alpha}^{2}$, which we also denote by $T_{f}^{\alpha} T_{\bar{g}}^{\alpha}$. Then, by (17) and Lemma 5.6, we have

$$
B_{\alpha}\left(|f|^{2}\right)\left(w_{n}\right) B_{\alpha}\left(|g|^{2}\right)\left(w_{n}\right)=\left\|\left(T_{f}^{\alpha} \tilde{k}_{w_{n}}^{\alpha}\right)\right\|^{2}\left\|\left(T_{g}^{\alpha} \tilde{k}_{w_{n}}^{\alpha}\right)\right\|^{2}=\left\|\left(1-M_{T_{\phi_{w_{n}}}^{\alpha}}\right)^{\alpha+2}\left(T_{f}^{\alpha} T_{\bar{g}}^{\alpha}\right)\right\|^{2},
$$

and the last term tends to zero for any sequence $w_{n}$ that converges to a point of the unit circle. But now, since

and

$$
|f(w)| \leq B_{\alpha}\left(|f|^{2}\right)(w)
$$

$$
|g(w)| \leq B_{\alpha}\left(|g|^{2}\right)(w)
$$

for every $w \in \mathbb{D}$, we can apply the classical result of Lusin and Priwaloff $[\mathrm{LP}$ to show that the analytic function $f g$ is identically equal to 0 . Thus, $f \equiv 0$ or $g \equiv 0$.

Remark. It follows immediately from Proposition 5.7 that the sum in Proposition 5.5 (convergent in the strong operator topology) can never be norm convergent, except in the trivial case where $T_{f} T_{\bar{g}}=0$.

\section{$\S 6$. THE SECOND THEOREM}

Our second result concerns a relationship between the boundedness of the product $B_{\beta}\left(|f|^{2}\right)(w) B_{\beta}\left(|g|^{2}\right)(w)$ and the boundedness of the operator $T_{f}^{\beta} T_{\bar{g}}^{\beta}$ acting on the spaces $A_{\alpha}^{2}$ for certain $\alpha<\beta$. As before, we begin with some estimates concerning Toeplitz operators and their derivatives.

Lemma 6.1. Let $\beta>\alpha>-1$, and let $f \in A_{\beta}^{2}$. Then for $w \in \mathbb{D}$ and $h \in L^{2}\left(\mathbb{D}, d A_{\alpha}\right)$ we have

$$
\left|\left(T \frac{\beta}{f} h\right)(w)\right| \leq \frac{1}{\left(1-|w|^{2}\right)^{1+\frac{\beta}{2}}}\left(B_{\beta}\left(|f|^{2}\right)(w)\right)^{\frac{1}{2}}\|h\|_{\alpha} .
$$

Proof. Suppose $w \in \mathbb{D}$ and $h \in A_{\alpha}^{2} \subseteq A_{\beta}^{2}$. Then, using the Hölder inequality, we obtain

$$
\left|\left(T \frac{\beta}{f} h\right)(w)\right|=\left\langle\bar{f} h, k_{w}^{\beta}\right\rangle_{\beta}=\left\langle h, f k_{w}^{\beta}\right\rangle_{\beta} \leq\|h\|_{\beta}\left\|f k_{w}^{\beta}\right\|_{\beta} .
$$

Now, since

we see that

$$
B_{\beta}\left(|f|^{2}\right)(w)=\left\|f \frac{k_{w}^{\beta}}{\left\|k_{w}^{\beta}\right\|_{\beta}}\right\|_{\beta}^{2}=\left(1-|w|^{2}\right)^{2+\beta}\left\|f k_{w}^{\beta}\right\|_{\beta}^{2}
$$

$$
\left|\left(T_{\bar{f}}^{\beta} h\right)(w)\right| \leq \frac{1}{\left(1-|w|^{2}\right)^{1+\frac{\beta}{2}}}\left(B_{\beta}\left(|f|^{2}\right)(w)\right)^{\frac{1}{2}}\|h\|_{\beta} .
$$

Since $\alpha \leq \beta$ implies $\|h\|_{\beta} \leq\|h\|_{\alpha}$, this finishes the proof. 
Next, we do an estimation for the values of the derivative of our Toeplitz operator.

Lemma 6.2. Suppose $\beta>-1, w \in \mathbb{D}$, and $h \in A_{\beta}^{2}$. Then

$$
\left|\left(T_{\bar{f}}^{\beta} h\right)^{\prime}(w)\right| \leq \frac{2+\beta}{1-|w|^{2}}\left(B_{\beta}\left(|f|^{2}\right)(w)\right)^{\frac{1}{2}}\left(P^{(-\beta, \beta)}\left(|h|^{2}\right)(w)\right)^{\frac{1}{2}} .
$$

Proof. We proceed as in the proof of Lemma 4.2 to see that

$$
\left|\left(T_{\bar{f}}^{\beta} h\right)^{\prime}(w)\right| \leq(2+\beta) \int_{\mathbb{D}} \frac{|f(z)||h(z)|}{|1-\bar{z} w|^{3+\beta}} d A_{\beta}(z) .
$$

Then, using Hölder's inequality along with the right decomposition of the product, we arrive at

$$
\begin{aligned}
\left|\left(T_{\frac{\beta}{f}}^{\beta} h\right)^{\prime}(w)\right| \leq(2+\beta)\left(\int_{\mathbb{D}} \frac{|f(z)|^{2}}{|1-\bar{z} w|^{4+2 \beta}} d A_{\beta}(z)\right)^{\frac{1}{2}}\left(\int_{\mathbb{D}} \frac{|h(z)|^{2}}{|1-\bar{z} w|^{2}} d A_{\beta}(z)\right)^{\frac{1}{2}} \\
\quad=\frac{(2+\beta)(1+\beta)}{\left(1-|w|^{2}\right)^{1+\frac{\beta}{2}}}\left(B_{\beta}\left(|f|^{2}\right)(w)\right)^{\frac{1}{2}}\left(\int_{\mathbb{D}}|h(z)|^{2} \frac{\left(1-|z|^{2}\right)^{\beta}}{|1-\bar{z} w|^{2}} d A(z)\right)^{\frac{1}{2}} \\
\quad=\frac{(2+\beta)(1+\beta)}{\left(1-|w|^{2}\right)}\left(B_{\beta}\left(|f|^{2}\right)(w)\right)^{\frac{1}{2}}\left(\int_{\mathbb{D}}|h(z)|^{2} \frac{\left(1-|z|^{2}\right)^{\beta}\left(1-|w|^{2}\right)^{-\beta}}{|1-\bar{z} w|^{2}} d A(z)\right)^{\frac{1}{2}} \\
\quad=\frac{(2+\beta)(1+\beta)}{\left(1-|w|^{2}\right)}\left(B_{\beta}\left(|f|^{2}\right)(w)\right)^{\frac{1}{2}}\left(P^{(-\beta, \beta)}\left(|h|^{2}\right)(w)\right)^{\frac{1}{2}} .
\end{aligned}
$$

Now we are ready to prove Theorem 2.4 .

Proof of Theorem 2.4. Let

$$
M=\sup _{w \in \mathbb{D}}\left(B_{\beta}\left(|f|^{2}\right)(w)\right)\left(B_{\beta}\left(|g|^{2}\right)(w)\right) .
$$

Precisely as in the proof of Theorem 2.2, it suffices to show that there exist absolute constants $K_{1}, K_{2}, K_{3}$ such that for any $u, v \in A_{\alpha}^{2}$ we have

$$
\begin{aligned}
\mathrm{I} & =\frac{\alpha+3}{\alpha+1} \int_{\mathbb{D}}\left(1-|w|^{2}\right)^{2}\left(T_{\bar{g}}^{\beta} u\right)(w) \overline{\left(T_{\frac{\beta}{f}}^{\beta} v\right)(w)} d A_{\alpha}(w) \\
& \leq K_{1}\|u\|_{\alpha}\|v\|_{\alpha}, \\
\mathrm{II} & =\frac{1}{(\alpha+1)(\alpha+2)} \int_{\mathbb{D}}\left(1-|w|^{2}\right)^{2}\left(T_{\bar{g}}^{\beta} u\right)^{\prime}(w) \overline{\left(T_{\frac{\beta}{f}}^{\beta} v\right)^{\prime}(w)} d A_{\alpha}(w) \\
& \leq K_{2}\|u\|_{\alpha}\|v\|_{\alpha}, \\
\mathrm{III} & =\frac{1}{(\alpha+1)(\alpha+3)} \int_{\mathbb{D}}\left(1-|w|^{2}\right)^{3}\left(T_{\bar{g}}^{\beta} u\right)^{\prime}(w) \overline{\left(T_{\bar{f}}^{\beta} v\right)^{\prime}(w)} d A_{\alpha}(w) \\
& \leq K_{3}\|u\|_{\alpha}\|v\|_{\alpha} .
\end{aligned}
$$

Applying Lemma 6.1, we see that there exists $K_{1}>0$ with

$$
\begin{aligned}
|\mathrm{I}| & \leq \frac{\alpha+3}{\alpha+1} \int_{\mathbb{D}}\left(1-|w|^{2}\right)^{2} \frac{1}{\left(1-|w|^{2}\right)^{2+\beta}}\left(B_{\beta}\left(|f|^{2}\right)(w)\right)^{\frac{1}{2}} \\
& \quad \times\left(B_{\beta}\left(|g|^{2}\right)(w)\right)^{\frac{1}{2}}\|u\|_{\alpha}\|v\|_{\alpha} d A_{\alpha}(w) \\
& \leq \frac{(\alpha+3)(\alpha+1)}{\alpha+1} M^{\frac{1}{2}}\|u\|_{\alpha}\|v\|_{\alpha} \int_{\mathbb{D}} \frac{1}{\left(1-|w|^{2}\right)^{\beta-\alpha}} d A(w) \\
& \leq K_{1}\|u\|_{\alpha}\|v\|_{\alpha} .
\end{aligned}
$$


Applying Lemma 6.2 and Hölder's inequality, we obtain

$$
\begin{aligned}
|\mathrm{II}| \leq & \frac{(\beta+2)^{2}}{(\alpha+1)(\alpha+2)} \int_{\mathbb{D}}\left(B_{\beta}\left(|f|^{2}\right)(w)\right)^{\frac{1}{2}}\left(B_{\beta}\left(|g|^{2}\right)(w)\right)^{\frac{1}{2}} \\
\leq & \times\left(P^{(-\beta, \beta)}\left(|u|^{2}\right)(w)\right)^{\frac{1}{2}}\left(P^{(-\beta, \beta)}\left(|v|^{2}\right)(w)\right)^{\frac{1}{2}} d A_{\alpha}(w) \\
\leq & \frac{(\beta+2)^{2}}{(\alpha+1)(\alpha+2)} M^{\frac{1}{2}}\left(\int_{\mathbb{D}} P^{(-\beta, \beta)}\left(|u|^{2}\right)(w) d A_{\alpha}(w)\right)^{1 / 2} \\
& \times\left(\int_{\mathbb{D}} P^{(-\beta, \beta)}\left(|v|^{2}\right)(w) d A_{\alpha}(w)\right)^{1 / 2} .
\end{aligned}
$$

Now, applying Theorem 3.2 with $p=1, a=-\beta$, and $b=\beta$, we see that $P^{-\beta, \beta}$ is a bounded operator on $L^{1}\left(\mathbb{D}, d A_{\alpha}\right)$, and so there exists $K_{2}>0$ such that

$$
|\mathrm{II}| \leq \frac{(\beta+2)^{2}}{(\alpha+1)(\alpha+2)} M^{1 / 2}\left\|P^{-\beta, \beta}\right\|_{L_{\alpha}^{1} \rightarrow L_{\alpha}^{1}}\|u\|_{\alpha}\|v\|_{\alpha} \leq K_{2}\|u\|_{\alpha}\|v\|_{\alpha}
$$

Finally, since $\left(1-|w|^{2}\right)^{3} \leq\left(1-|w|^{2}\right)^{2}$ for any $w$ in $\mathbb{D}$, we also have

$$
|\mathrm{III}| \leq K_{2}\|u\|_{\alpha}\|v\|_{\alpha} .
$$

This finishes the proof.

\section{§7. REMARK}

In the case of invertible products of Toeplitz operators on $A_{0}^{2}$, Stroethoff and Zheng showed that Sarason's condition (5) is indeed sufficient [StZh2]. Recently, M. Smith has obtained the corresponding result for the weighted Bergman spaces $A_{\alpha}^{2}, \alpha>-1$. Smith has also obtained a necessary and a sufficient condition for the boundedness of product Toeplitz operators on unweighted $p$-Bergman $A^{p}$ space for $1<p<\infty$; see $\underline{\mathrm{Sm}}$.

\section{REFERENCES}

[HaNik] V. P. Havin and N. K. Nikolski, Stanislav Aleksandrovich Vinogradov, his life and mathematics, Complex Analysis, Operators, and Related Topics, Oper. Theory Adv. Appl., vol. 113, Birkhäuser, Basel, 2000, pp. 1-18. MR1771747 (2001b:01025a)

[HKZ] H. Hedenmalm, B. Korenblum, and K. Zhu, Theory of Bergman spaces, Grad. Texts in Math., vol. 199, Springer-Verlag, New York, 2000. MR1758653 (2001c:46043)

[Lu] D. Luecking, Forward and reverse Carleson inequalities for functions in Bergman spaces and their derivatives, Amer. J. Math. 107 (1985), 85-111. MR0778090 (86g:30002)

[LP] N. Lusin and J. Priwaloff, Sur l'unicité et la multiplicité des fonctions analytiques, Ann. Sci. École Norm. Sup. (3) 42 (1925), no. 3, 143-191.

[M] Mathworld Website http://mathworld.wolfram.com/BetaFunction.html

[N1] F. Nazarov, A counterexample to Sarason's conjecture, Preprint, www.math.msu.edu/ fedja/pepr.html

[N2] F. Nazarov, Private communication.

[S] D. Sarason, Products of Toeplitz operators, Linear and Complex Analysis. Problem Book 3, Part I (V. P. Havin and N. K. Nikolski, eds.), Lecture Notes in Math., vol. 1573, Springer-Verlag, Berlin, 1994.

[Sm] M. Smith, Preprint, 2004.

[StZh1] K. Stroethoff and D. Zheng, Products of Hankel and Toeplitz operators on the Bergman space, J. Funct. Anal. 169 (1999), 289-313. MR1726756 (2000i:47053)

[StZh2] _ Invertible Toeplitz products, J. Funct. Anal. 195 (2002), no. 1, 48-70. MR1934352 (2003g:47051)

[TVZh] S. Treil, A. Volberg, and D. Zheng, Hilbert transform, Toeplitz operators and Hankel operators, and invariant $A_{\infty}$ weights, Rev. Mat. Iberoamericana 13 (1997), no. 2, 319-360. MR.1617653 (2000e:47061) 
[VMü] V. Müller and F.-H. Vasilescu, Standard models for some commuting multioperators, Proc. Amer. Math. Soc. 117 (1993), no. 4, 979-989. MR1112498 (93e:47016)

[Zh] D. Zheng, The distribution function inequality and products of Toeplitz operators and Hankel operators, J. Funct. Anal. 138 (1996), no. 2, 477-501. MR.1395967 (97e:47040)

Department of Mathematics, University of Glasgow, Glasgow G12 8QW, United Kingdom

E-mail address: sp@maths.gla.ac.uk

Departement de Mathématiques Pures, Université Bordeaux I, 351, Cours de la Libération, 33405 Talence Cedex, France

E-mail address: Elizabeth.Strouse@math.u-bordeaux1.fr

Received 3/OCT/2005

Originally published in English 